\title{
Các nhân tố quyết định năng lực giảng dạy của sinh viên sư phạm: Khảo sát tại trường Đại học Sư phạm Thành phố Hồ Chí Minh
}

\section{Factors effecting the teaching capacity of pedagogical students: Survey at Ho Chi Minh City University of Education}

\author{
Lê Thái Minh Long ${ }^{1 *}$ \\ ${ }^{1}$ Trường Đại học Khoa học Xã hội và Nhân văn, ĐHQG Thành phố Hồ Chí Minh, Việt Nam \\ *Tác giả liên hệ, Email: longltm9498@gmail.com
}

THÔNG TIN

DOI: $10.46223 / \mathrm{HCMCOUJS.}$ soci.vi.16.2.1949.2021

Ngày nhận: 20/06/2021

Ngày nhận lại: 08/08/2021

Duyệt đăng: 14/08/2021

Tù khóa:

kế hoạch dạy học; năng lực giảng dạy; phương pháp dạy học; sinh viên sư phạm

Keywords:

teaching plan; teaching capacity; teaching method; pedagogical students

\section{TÓM TĂT}

Xác định các nhân tố quyết định năng lực giảng dạy là mục tiêu quan trọng mà các trường Đại học Sư phạm cần đảm bảo để sinh viên khi tốt nghiệp ra trường có thể giảng dạy được ở các trường phổ thông. Bài nghiên cứu sử dụng kiểm định số liệu thống kê, phân tích hồi quy để xác định các thành tố tác động đến năng lực giảng dạy của sinh viên sư phạm tại Trường Đại học Sư phạm Thành phố Hồ Chí Minh. Các nhân tố tác động năng lực giảng dạy trong nghiên cứu bao gồm các thành tố: xây dựng kế hoạch dạy học, sử dụng phương pháp dạy học, kiểm tra, đánh giá kết quả học tập, phát triển chuyên môn bản thân, tư vấn và hỗ trợ học sinh, mỗi thành tố với các mức độ tác động khác nhau tạo thành năng lực giảng dạy đối với sinh viên sư phạm. Các nhân tố này là cơ sở cho các trường đại học sư phạm xây dựng chương trình đào tạo, phân bố thời gian hợp lí để rèn luyện các năng lực giảng dạy cho sinh viên sư phạm.

\section{ABSTRACT}

To ensure that graduates can teach in high schools, pedagogical students must be guaranteed that their teaching capacity is high quality. The factors affecting the teaching capacity of pedagogical students at the Ho Chi Minh University of Education are investigated using statistical tests and regression analysis. The factors affecting the teaching capacity of pedagogical students includes the following cities: developing teaching plans, using teaching methods, testing, assessing learning outcomes, developing personal expertise, consulting, advising, and supporting students, each of which has varying degrees of impact on pedagogical students' teaching capacity. These factors are the basis for education universities to develop training programs and allocate time appropriately to train teaching capacities for pedagogical students. 


\section{Giới thiệu}

Nghị quyết Hội nghị lần thứ VIII Ban chấp hành Trung ương khóa XI đã chỉ rõ một trong những nhiệm vụ, giải pháp để phát triển giáo dục, đó là: "Phát triển đội ngũ nhà giáo và cán bộ quản lý, đáp ứng nhu cầu đổi mới giáo dục đào tạo" (Ban chấp hành Trung ương Đảng Cộng sản Việt Nam, 2013, p. 8). Trong Nghị quyết Đại hội đại biểu toàn quốc lần thứ XIII của Đảng có nêu cần phải: "Tạo đột phá trong đổi mới căn bản, toàn diện giáo dục và đào tạo, phát triển nguồn nhân lực chất lượng cao" (Ban chấp hành Trung ương Đảng Cộng sản Việt Nam, 2021, p. 5) và "Tạo bước chuyển biến mạnh mẽ, toàn diện, cơ bản về chất lượng giáo dục, đào tạo gắn với cơ chế tuyển dụng, sử dụng, đãi ngộ nhân tài” (Ban chấp hành Trung ương Đảng Cộng sản Việt Nam, 2021, p. 9). Những quan tâm của Đảng quan tâm và trình bày trong các nghị quyết nêu trên cho thấy vai trò của giáo dục ngày một quan trọng hơn, đòi hỏi đội ngũ giảng dạy không ngừng bồi dưỡng, nâng cao năng lực để đáp ứng nhu cầu đổi mới giáo dục ở Việt Nam hiện nay.

Chương trình giáo dục phổ thông năm 2018 được Bộ Giáo dục và Đào tạo ban hành với rất nhiều yêu cầu cần đạt về phát triển phẩm chất và năng lực của học sinh (Bộ Giáo dục và Đào tạo, 2018a). Chương trình được xây dựng theo định hướng của xu thế các nước trên thế giới trong thiết kế chương trình giáo dục phổ thông của quốc gia theo hướng tiếp cận năng lực và được xem là chuẩn đầu ra (Bui, Dao, \& Hoang, 2012), giáo viên cần phải tích lũy kiến thức chuyên môn khoa học, bồi dưỡng kĩ năng và nghiệp vụ sư phạm từ đó hình thành năng lực giảng dạy để tiếp cận được sự đổi mới trong giáo dục hiện nay, từ đó, đặt ra yêu cầu thực hiện nghiêm túc và đầy đủ việc nâng cao chất lượng đội ngũ và đổi mới cơ chế, chính sách dành cho việc bồi dưỡng thường xuyên cho giáo viên, đây là điều luôn thu hút sự quan tâm không chỉ đối với ngành giáo dục và đào tạo mà còn đối với toàn xã hội (Le, 2019).

Chất lượng năng lực giảng dạy là mục tiêu quan trọng mà các trường đại học sư phạm cần đảm bảo để sinh viên khi tốt nghiệp ra trường có thể giảng dạy được ở các trường phổ thông (D. T. Nguyen, 2013). Sinh viên sư phạm tự phát triển năng lực giảng dạy là một hoạt động có ý thức, chủ động trong việc nắm vững kiến thức chuyên môn, kiến thức sư phạm và rèn luyện chuyên môn nghiệp vụ giảng dạy thường xuyên trong quá trình được đào tạo tại trường sư phạm (Gebre, Saroyan, \& Bracewell, 2014; Mirzagitova \& Akhmetov, 2015). Quá trình truyền đạt lí thuyết về giảng dạy và rèn luyện năng lực giảng dạy cho sinh viên sư phạm trong quá trình đào tạo tại trường đại học giúp cho sinh viên sư phạm có khả năng chuẩn bị và áp dụng hiệu quả vào các bài dạy trong tương lai khi trở thành giáo viên (Panagiota \& Eleni, 2021). Tuy nhiên, các nghiên cứu còn hạn chế trong việc phân tích các năng lực giảng dạy cần hình thành cho sinh viên sư phạm trong quá trình đào tạo tại trường, vì thế sinh viên sư phạm không nắm bắt được các năng lực giảng dạy cần hình thành để có thể rèn luyện một cách hợp lí và đầy đủ.

Từ những lí do trên, đề tài khảo sát năng lực của sinh viên trong giảng dạy, thông qua thống kê và phân tích để xác định cấu trúc năng lực giảng dạy của sinh viên tại Trường Đại học Sư phạm Thành phố Hồ Chí Minh từ đó phân tích các nhân tố ảnh hưởng. Các nhân tố quyết định năng lực giảng dạy này là cơ sở để trường định hướng và có kế hoạch rèn luyện năng lực giảng dạy cho sinh viên một cách hiệu quả hơn.

\section{Cơ sở lý thuyết}

Tác giả Trịnh Thúy Giang và Mai Quốc Khánh đã định nghĩa về năng lực dạy học như sau "Năng lực dạy học là sự thực hiện thành công một nhiệm vụ dạy học hoặc giải quyết hiệu quả những vấn đề đặt ra của thực tiễn dạy học, trên cơ sở vận dụng hợp lí, linh hoạt, mềm dẻo, sáng tạo những kiến thức, kĩ năng chuyên môn và chuẩn thái độ nghề nghiệp tương ứng" (Trinh \& Mai, 2019, p. 35). Sinh viên sư phạm tại các trường Đại học Sư phạm phải được rèn luyện năng lực giảng dạy một cách bài bản và đầy đủ nhất để trở thành một giáo viên thực hiện công 
tác giảng dạy trong tương lai. Do đó, để trở thành một giáo viên, mục tiêu và năng lực giảng dạy của sinh viên sư phạm cần đạt được phải được các trường đại học sư phạm xác định rõ và đầy đủ trong các chương trình đào tạo giáo viên (Fielding \& Schalock, 1985).

Chương trình được xây dựng hướng đến mục tiêu "chuyển biến căn bản, toàn diện về chất lượng và hiệu quả giáo dục phổ thông; kết hợp dạy chữ, dạy người và định hướng nghề nghiệp; góp phần chuyển nền giáo dục nặng về truyền thụ kiến thức sang nền giáo dục khuyến khích phát triển toàn diện cả về phẩm chất và năng lực, hài hòa đức, trí, thể, mỹ và phát huy tốt nhất tiềm năng của mỗi học sinh" (Bộ Giáo dục và Đào tạo, 2018a, p. 3), quan điểm được thể hiện xuyên suốt chương trình, là một trong những mục tiêu quan trọng của chương trình là "bảo đảm phát triển phẩm chất và năng lực người học thông qua nội dung giáo dục với những kiến thức, kĩ năng cơ bản, thiết thực, hiện đại” (Bộ Giáo dục và Đào tạo, 2018a, p. 5).

Năng lực giảng dạy cần được hình thành thông qua con đường thực hành, luyện tập thường xuyên, đòi hỏi chương trình đào tạo và môi trường giáo dục tại trường Đại học Sư phạm phải tạo môi trường học tập để người học có cơ hội hình thành năng lực giảng dạy một cách hiệu quả được (H. T. T. Nguyen, 2015). Shulman (1987) đưa ra các điều kiện tiên quyết của việc dạy học hiệu quả là: kiến thức chuyên môn, kiến thức giáo dục chung, kiến thức chương trình giảng dạy, kiến thức phương pháp dạy học, kiến thức tìm hiểu về người học, kiến thức về bối cảnh giáo dục, kiến thức về mục đích giáo dục. Trong đó, kiến thức về sư phạm và phương pháp dạy học là hai yếu tố quan trọng nhất trong năng lực giảng dạy. Nếu thiếu hai kiến thức này thì quá trình dạy học sẽ không được diễn ra theo kế hoạch đã định và không thể truyền đạt kiến thức môn học cho học sinh. Voss, Kunter, và Baumert (2011) và nghiên cứu của Hammond (2006) đã đưa ra các năng lực cơ bản của mỗi giáo viên: năng lực quản lý lớp học, năng lực sử dụng phương pháp dạy học, năng lực thực hiện quá trình truyền tải kiến thức đến cho học sinh, năng lực kiểm tra, đánh giá học sinh, và năng lực nhận diện đặc điểm từng cá nhân học sinh.

Trong quá trình đào tạo tại trường, sinh viên sư phạm được rèn luyện nhiều năng lực khác nhau để đạt tiêu chuẩn nghề nghiệp của một giáo viên. Bộ Giáo dục và Đào tạo đã ban hành các tiêu chuẩn giáo viên gồm 05 tiêu chuẩn cụ thể: tiêu chuẩn 1 - phẩm chất nhà giáo; tiêu chuẩn 2 - phát triển chuyên môn, nghiệp vụ; tiêu chuẩn 3 - xây dựng môi trường giáo dục; tiêu chuẩn 4 - phát triển mối quan hệ giữa nhà trường, gia đình và xã hội; tiêu chuẩn 5 - sử dụng ngoại ngữ hoặc tiếng dân tộc, ứng dụng công nghệ thông tin, khai thác và sử dụng thiết bị công nghệ trong dạy học, giáo dục (Bộ Giáo dục và Đào tạo, 2018b, pp. 3-7). Năng lực giảng dạy thuộc nội dung của tiêu chuẩn 2 - phát triển chuyên môn, nghiệp vụ, với các năng lực cụ thể: phát triển chuyên môn bản thân; xây dựng kế hoạch dạy học và giáo dục theo hướng phát triển phẩm chất, năng lực học sinh; sử dụng phương pháp dạy học và giáo dục theo hướng phát triển phẩm chất, năng lực học sinh; kiểm tra, đánh giá theo hướng phát triển phẩm chất, năng lực học sinh; tư vấn và hỗ trợ học sinh.

Theo tác giả Đinh Quang Báo, năng lực giảng dạy bao gồm: tìm hiểu, chuẩn đoán nhu cầu của học sinh, năng lực thiết kế kế hoạch dạy học, năng lực thực hiện kế hoạch dạy học, năng lực giám sát kiểm tra, đánh giá, năng lực giải quyết các vấn đề phát sinh trong dạy học (Dinh, 2013). Thông qua các cơ sở lý luận đã được trình bày trên, trong nghiên cứu này, năng lực giảng dạy của sinh viên sư phạm được xác định gồm: Phát triển chuyên môn bản thân, Xây dựng kế hoạch dạy học, Sử dụng phương pháp dạy học, Kiểm tra, đánh giá kết quả học tập, Tư vấn và hỗ trợ học sinh. Mỗi năng lực cấu trúc thành năng lực giảng dạy của sinh viên được xem là một thành phần (nhân tố) với các tiêu chí quan sát (biến quan sát), từ đó xem xét ảnh hưởng của các nhân tố cấu trúc nên năng lực giảng dạy của sinh viên sư phạm, được xem xét thông qua sinh viên sư phạm tại Trường Đại học Sư phạm Thành phố Hồ Chí Minh. 


\section{Bảng 1}

Cấu trúc các nhân tố quyết định năng lực giảng dạy của sinh viên sư phạm

\begin{tabular}{|c|c|c|}
\hline \multirow{23}{*}{$\begin{array}{c}\text { Năng lực giảng } \\
\text { dạy của sinh } \\
\text { viên sư phạm tại } \\
\text { Trường Đại học } \\
\text { Sư phạm } \\
\text { Thành phố Hồ } \\
\text { Chí Minh }\end{array}$} & \multirow{5}{*}{$\begin{array}{l}\text { Phát triển chuyên } \\
\text { môn bản thân }\end{array}$} & Năng lực chuyên môn khoa học \\
\hline & & Năng lực về lí luận và phương pháp dạy học \\
\hline & & Năng lực tìm hiểu về chương trình dạy học \\
\hline & & Năng lực sư phạm thực tiễn \\
\hline & & Hướng dẫn, hỗ trợ và chia sẻ chuyên môn bản thân \\
\hline & & Kế hoạch dạy học trình bày theo quy định chung \\
\hline & & $\begin{array}{l}\text { Xác định mục tiêu dạy học phát triển phẩm chất, } \\
\text { năng lực học sinh }\end{array}$ \\
\hline & $\begin{array}{l}\text { Xây dựng kế hoạch } \\
\text { dạy học }\end{array}$ & $\begin{array}{l}\text { Nội dung dạy học bám sát chương trình, hướng tới } \\
\text { phát triển phẩm chất, năng lực học sinh }\end{array}$ \\
\hline & & Hoạt động dạy học gắn với thực tiễn \\
\hline & & $\begin{array}{l}\text { Hướng dẫn, hỗ trợ và chia sẻ kinh nghiệm xây dựng } \\
\text { kế hoạch dạy học }\end{array}$ \\
\hline & & $\begin{array}{l}\text { Cập nhật được những phương pháp dạy học tích } \\
\text { cực phát triển phẩm chất, năng lực học sinh }\end{array}$ \\
\hline & Sử dl & $\begin{array}{l}\text { Vận dụng những phương pháp dạy học tích cực } \\
\text { phát triển phẩm chất, năng lực học sinh }\end{array}$ \\
\hline & phóndarbo & $\begin{array}{l}\text { Phối hợp các phương pháp dạy học tích cực và các } \\
\text { phương pháp dạy học truyền thống trong một kế } \\
\text { hoạch bài dạy }\end{array}$ \\
\hline & & Hướng dẫn, hỗ trợ và chia sẻ phương pháp dạy học \\
\hline & & $\begin{array}{l}\text { Cập nhật được những hình thức kiểm tra, đánh giá } \\
\text { phát triển phẩm chất, năng lực học sinh }\end{array}$ \\
\hline & & $\begin{array}{l}\text { Vận dụng được nhiều hình thức kiểm tra, đánh giá } \\
\text { trong kế hoạch dạy học }\end{array}$ \\
\hline & $\begin{array}{l}\text { Kiểm tra, đánh giá } \\
\text { kêt quả học tập }\end{array}$ & $\begin{array}{l}\text { Kiểm tra, đánh giá kết quả học tập của học sinh } \\
\text { đảm bảo chính xác, toàn diện }\end{array}$ \\
\hline & & $\begin{array}{l}\text { Sử dụng kết quả kiểm tra, đánh giá để điều chỉnh } \\
\text { hoạt động dạy học }\end{array}$ \\
\hline & & $\begin{array}{l}\text { Hướng dẫn, hỗ trợ và chia sẻ kinh nghiệm kiểm tra, } \\
\text { đánh giá kết quả học tập }\end{array}$ \\
\hline & & $\begin{array}{l}\text { Hiểu được phẩm chất, năng lực của học sinh trong } \\
\text { từng lớp học để có hỗ trợ kịp thời }\end{array}$ \\
\hline & Tư vấn và hỗ trơ & $\begin{array}{l}\text { Thực hiện các phương pháp hỗ trợ trong quá trình } \\
\text { dạy học, xử lí kịp thời các tình huống phát sinh } \\
\text { trong quá trình dạy học }\end{array}$ \\
\hline & học sinh & $\begin{array}{l}\text { Hỗ trợ học sinh trong quá trình giải quyết vấn đề } \\
\text { trong kế hoạch dạy học phát triến phâm chất, năng } \\
\text { lực học sinh }\end{array}$ \\
\hline & & $\begin{array}{l}\text { Chia sẻ kinh nghiệm hỗ trợ học sinh trong quá trình } \\
\text { giảng dạy }\end{array}$ \\
\hline
\end{tabular}

Nguồn: Kết quả xử lý dữ liệu của nhóm tác giả

\section{Phương pháp nghiên cứu}

Quy ước thang đo: bảng hỏi của nghiên cứu được sử dụng thang đo 05 mức độ để ánh giá các mức độ năng lực của sinh viên với 1 = "không đạt", 2 = "trung bình"; 3 ="khá"; 4 = "tốt"; 5 = "xuất sắc" về: phát triển chuyên môn bản thân, xây dựng kế hoạch dạy học, sử dụng phương 
pháp dạy học, kiểm tra đánh giá kết quả học tập, tư vấn hỗ trợ học sinh, năng lực giảng dạy chung của sinh viên.

Mẫu nghiên cứu: về các ngành đào tạo sư phạm tại Trường Đại học Sư phạm Thành phố Hồ Chí Minh, đề tài lựa chọn mẫu nghiên cứu phi ngẫu nhiên thuận tiện khi khảo sát sinh viên của 03 ngành về khối Khoa học tự nhiên là sư phạm Vật lý, sư phạm Hóa học và sư phạm Sinh học. Đề tài tiến hành khảo sát trên mẫu tổng thể chung sinh viên các ngành được lựa chọn với 180 sinh viên sư phạm của các ngành: sư phạm Vật lý, sư phạm Hóa học, sư phạm Sinh học với mỗi ngành 60 sinh viên trong đó có 30 sinh viên năm 04 và 30 sinh viên năm 03 để thực hiện khảo sát, thời gian khảo sát vào tháng $05 / 2021$ sau khi sinh viên năm 03 và năm 04 đã hoàn thành thực tập sư phạm nhằm đánh giá năng lực giảng dạy của sinh viên có được.

Phương pháp phân tích dữ liệu: kết quả nghiên cứu của đề tài được sử dụng phương pháp thống kê toán học bằng phần mềm IBM SPSS Statistic 22.0 để phân tích số liệu, kiểm định mô hình, phân tích hồi quy và đưa ra kết quả nghiên cứu. Kết quả nghiên cứu là cơ sở để đưa ra cấu trúc năng lực giảng dạy của sinh viên sư phạm tại Trường Đại học Sư phạm Thành phố Hồ Chí Minh.

\section{Kết quả nghiên cứu và thảo luận}

\subsection{Kết quả độ tin cậy của thang đo năng lục giảng dạy của sinh viên sư phạm}

Năng lực giảng dạy trong nghiên cứu được đề xuất với 05 thành phần và 23 biến quan sát. Đề tài sử dụng hệ số Cronbach's alpha và hệ số tương quan biến tổng làm cơ sở đánh giá độ tin cậy của thanh đo năng lực giảng dạy của sinh viên sư phạm được khảo sát trong nghiên cứu. Cronbach (1951) đưa ra hệ số tin cậy cho thang đo, với hệ số Cronbach's alpha là một phép toán kiểm định thống kê về sự chặt chẽ mà các mục câu hỏi trong thang đo tương quan với nhau và hệ số tương quan biến tổng thể hiện sự tương quan chặt chẽ các biến để đo lường cùng một khái niệm nghiên cứu. Hệ số Cronbach's alpha được sử dụng để đo lường độ tin cậy của thang đo gồm từ 03 biến quan sát trở lên và so sánh, phân tích sự tương quan của từng biến đến cả thang đo, hệ số không tính độ tin cậy cho từng biến quan sát trong thang đo. Theo Hair, Tatham, Anderson, và Black (2009), hệ số Cronbach's alpha $\geq 0.6$ là thang đo có giá trị có thể sử dụng và Nunnally (1978) cho rằng thang đo có giá trị tin cậy cao với giá trị từ 0.7 trở lên. Biến quan sát có chỉ số tương quan biến tổng $\geq 0.3$ thì biến đó đạt yêu cầu trong nghiên cứu. Khi sử dụng SPSS để phân tích tương quan biến tổng ta cũng cần chú ý đến giá trị của cột Cronbach's alpha if Item Deleted, cột này biểu diễn hệ số Cronbach's alpha nếu loại biến đang xem xét. Nếu hệ số Cronbach's alpha thấp làm thang đo chưa đủ độ tin cậy thì có thể xem xét loại biến quan sát có chỉ số tương quan biến tổng bé hơn 0.3 , phương pháp này nhằm loại bỏ các biến không phù hợp. Kết quả phân tích năng lực giảng dạy của sinh viên sư phạm được trình bày ở Bảng 2 .

\section{Bảng 2}

Mức độ tin cậy của thang đo năng lực giảng dạy của sinh viên sư phạm

\begin{tabular}{|c|c|c|c|}
\hline & Nhân tố quan sát & $\begin{array}{c}\text { Tưong } \\
\text { quan biến } \\
\text { tổng }\end{array}$ & $\begin{array}{c}\text { Cronbach's } \\
\text { alpha }\end{array}$ \\
\hline \multirow{3}{*}{$\begin{array}{l}\text { Phát triển chuyên } \\
\text { môn bản thân }\end{array}$} & Năng lực chuyên môn khoa học & 0.400 & \multirow{3}{*}{0.707} \\
\hline & $\begin{array}{l}\text { Năng lực về lí luận và phương pháp dạy } \\
\text { học }\end{array}$ & 0.858 & \\
\hline & Năng lực tìm hiểu về chương trình dạy & 0.899 & \\
\hline
\end{tabular}




\begin{tabular}{|c|c|c|c|}
\hline & Nhân tố quan sát & $\begin{array}{c}\text { Tương } \\
\text { quan biến } \\
\text { tổng }\end{array}$ & $\begin{array}{c}\text { Cronbach's } \\
\text { alpha }\end{array}$ \\
\hline & học & & \\
\hline & Năng lực sư phạm thực tiễn & 0.665 & \\
\hline & $\begin{array}{l}\text { Hướng dẫn, hỗ trợ và chia sẻ chuyên môn } \\
\text { bản thân }\end{array}$ & 0.812 & \\
\hline & $\begin{array}{l}\text { Kế hoạch dạy học trình bày theo quy định } \\
\text { chung }\end{array}$ & 0.527 & \\
\hline & $\begin{array}{l}\text { Xác định mục tiêu dạy học phát triển } \\
\text { phẩm chất, năng lực học sinh }\end{array}$ & 0.899 & \\
\hline $\begin{array}{l}\text { Xây dựng kế hoạch } \\
\text { dạy học }\end{array}$ & $\begin{array}{l}\text { Nội dung dạy học bám sát chương trình, } \\
\text { hướng tới phát triển phẩm chất, năng lực } \\
\text { học sinh }\end{array}$ & 0.782 & 0.889 \\
\hline & Hoạt động dạy học gắn với thực tiễn & 0.867 & \\
\hline & $\begin{array}{l}\text { Hướng dẫn, hỗ trợ và chia sẻ kinh nghiệm } \\
\text { xây dựng kế hoạch dạy học }\end{array}$ & 0.853 & \\
\hline & $\begin{array}{l}\text { Cập nhật được những phương pháp dạy } \\
\text { học tích cực phát triển phẩm chất, năng } \\
\text { lực học sinh }\end{array}$ & 0.771 & \\
\hline Sử dụng phương & $\begin{array}{l}\text { Vận dụng những phương pháp dạy học } \\
\text { tích cực phát triển phẩm chất, năng lực } \\
\text { học sinh }\end{array}$ & 0.849 & 0800 \\
\hline pháp dę & $\begin{array}{l}\text { Phối hợp các phương pháp dạy học tích } \\
\text { cực và các phương pháp dạy học truyền } \\
\text { thống trong một kế hoạch bài dạy }\end{array}$ & 0.706 & \\
\hline & $\begin{array}{l}\text { Hướng dẫn, hỗ trợ và chia sẻ phương } \\
\text { pháp dạy học }\end{array}$ & 0.809 & \\
\hline & $\begin{array}{l}\text { Cập nhật được những hình thức kiểm tra, } \\
\text { đánh giá phát triền phầm chât, năng lực } \\
\text { học sinh }\end{array}$ & 0.842 & \\
\hline $\begin{array}{l}\text { Kiểm tra, đánh giá } \\
\text { kết quả học tập }\end{array}$ & $\begin{array}{l}\text { Vận dụng được nhiều hình thức kiểm tra, } \\
\text { đánh giá trong kế hoạch dạy học }\end{array}$ & 0.490 & 0.850 \\
\hline & $\begin{array}{l}\text { Kiểm tra, đánh giá kết quả học tập của } \\
\text { học sinh đảm bảo chính xác, toàn diện }\end{array}$ & 0.893 & \\
\hline
\end{tabular}




\begin{tabular}{|c|c|c|c|}
\hline \multicolumn{2}{|r|}{ Nhân tố quan sát } & $\begin{array}{c}\text { Tương } \\
\text { quan biến }\end{array}$ & $\begin{array}{l}\text { Cronbach's } \\
\text { alpha }\end{array}$ \\
\hline & $\begin{array}{l}\text { Sử dụng kết quả kiểm tra, đánh giá để } \\
\text { điều chỉnh hoạt động dạy học }\end{array}$ & 0.886 & \\
\hline & $\begin{array}{l}\text { Hướng dẫn, hỗ trợ và chia sẻ kinh nghiệm } \\
\text { kiểm tra, đánh giá kết quả học tập }\end{array}$ & 0.836 & \\
\hline \multirow{4}{*}{$\begin{array}{l}\text { Tư vấn và hỗ trọ̆ } \\
\text { học sinh }\end{array}$} & $\begin{array}{l}\text { Hiểu được phẩm chất, năng lực của học } \\
\text { sinh trong từng lớp học để có hỗ trợ kịp } \\
\text { thời }\end{array}$ & 0.759 & \multirow{4}{*}{0.869} \\
\hline & $\begin{array}{l}\text { Thực hiện các phương pháp hỗ trợ trong } \\
\text { quá trình dạy học, xử lí kịp thời các tình } \\
\text { huống phát sinh trong quá trình dạy học }\end{array}$ & 0.762 & \\
\hline & $\begin{array}{l}\text { Hỗ trợ học sinh trong quá trình giải quyết } \\
\text { vấn đề trong kế hoạch dạy học phát triển } \\
\text { phẩm chất, năng lực học sinh }\end{array}$ & 0.852 & \\
\hline & $\begin{array}{l}\text { Chia sẻ kinh nghiệm hỗ trợ học sinh trong } \\
\text { quá trình giảng dạy }\end{array}$ & 0.713 & \\
\hline
\end{tabular}

Nguồn: Kết quả xử lí dữ liệu thống kê từ IBM SPSS Statistic 22.0

Các giá trị hệ số Cronbach’s alpha của các năng lực thành phần có giả trị từ 0.707 đến 0.889 trong bảng kết quả mức độ tin cậy của thang đo trong nghiên cứu này có giá trị lớn hơn ngưỡng 0.6 (Hair, Tatham, et al., 2009) và lớn hơn 0.7 (Nunnally, 1978). Kết quả thống kê cho thấy các năng lực thành phần trong nghiên cứu có độ tin cậy cao để sử dụng, đã thỏa đáng cho việc đánh giá năng lực giảng dạy cho sinh viên sư phạm. Giá trị tương quan biến tổng của các biến quan sát trong 05 thành phần năng lực giảng dạy của sinh viên sư phạm có kết quả từ 0.400 đến 0.899 . Các biến quan sát này có chỉ số tương quan biến tổng $\geq 0.3$ thì biến đó đạt yêu cầu trong nghiên cứu, cho thấy các biến có thể đóng góp vào các năng lực thành phần. Chỉ số Cronbach's alpha của các biến thành phẩn đều lớn hơn 0.7 và tương quan biến tổng lớn hơn 0.3 nên trong nghiên cứu này không trình bày đến việc loại biến quan sát và tất cả biến quan sát đều phù hợp. Điều đó cho thấy 23 biến quan sát làm thành tố cấu tạo thành 05 thành phần năng lực hoàn toàn phù hợp để đại diện cho năng lực giảng dạy của sinh viên sư phạm từ kết quả nghiên cứu thu được từ sinh viên sư phạm tại Trường Đại học Sư phạm Thành phố Hồ Chí Minh.

\subsection{Kết quả phân tích nhân tố khám phá năng lục giảng dạy của sinh viên sư phạm}

Thông qua kiểm định chất lượng của thang đo Cronbach's alpha thì các biến quan sát đã phù hợp và đảm bảo tin cậy của thang đo. Sau khi kiểm định độ tin cậy Cronbach's alpha, tất cả các biến được đưa vào phân tích nhân tố khám phá để đánh giá mức độ hội tụ của các biến quan sát theo các thành phần nhằm đảm bảo giá trị phân biệt cho các thang đo. Trong phân tích nhân tố khám phá năng lực giảng dạy của sinh viên sư phạm, đè tài phân tích hệ số KMO (KaiserMeyer-Olkin), hệ số tải nhân tố và tổng phương sai trích. Hệ số KMO là một chỉ số dùng để xem xét sự thích hợp của phân tích nhân tố. Trị số của KMO phải đạt giá trị 0.5 trở lên $(0.5 \leq \mathrm{KMO} \leq$ 01) là điều kiện đủ để phân tích nhân tố là phù hợp. Nếu trị số này nhỏ hơn 0.5 , thì phân tích nhân tố có khả năng không thích hợp với tập dữ liệu nghiên cứu. Hệ số tải nhân tố hay còn gọi là 
trọng số nhân tố, giá trị này biểu thị mối quan hệ tương quan giữa biến quan sát với nhân tố. Hệ số tải nhân tố càng cao, nghĩa là tương quan giữa biến quan sát đó với nhân tố càng lớn và ngược lại. Giá trị chuẩn của hệ số tải nhân tố được xem xét cùng với số lượng mẫu thu được từ khảo sát. Số lượng mẫu nghiên cứu của đề tài thu được là 180 vì thế lựa chọn hệ số tải nhân tố $\geq 0.4$ và hệ số tải nhân tố $\geq 0.5$ thì biến quan sát có ý nghĩa thống kê tốt (Hair, Black, Babin, \& Anderson, 2009). Tổng phương sai trích $\geq 50 \%$ cho thấy mô hình phân tích nhân tố là phù hợp (Hair, Tatham, et al., 2009). Coi biến thiên là $100 \%$ thì trị số này thể hiện các nhân tố được trích cô đọng được bao nhiêu \% và bị thất thoát bao nhiêu \% của các thành phần (Hair, Black, et al., 2009), kết quả phân tích được trình bày ở Bảng 3 .

\section{Bảng 3}

Phân tích nhân tố khám phá năng lực giảng dạy của sinh viên sư phạm

\begin{tabular}{|c|c|c|c|}
\hline & Nhân tố quan sát & $\begin{array}{l}\text { Hệ số tải } \\
\text { nhân tố }\end{array}$ & $\begin{array}{c}\text { Tổng } \\
\text { phương sai } \\
\text { trích } \\
(\%)\end{array}$ \\
\hline \multirow{5}{*}{$\begin{array}{l}\text { Phát triển chuyên } \\
\text { môn bản thân }\end{array}$} & Năng lực chuyên môn khoa học & 0.501 & \multirow{5}{*}{65.05} \\
\hline & $\begin{array}{l}\text { Năng lực về lí luận và phương pháp dạy } \\
\text { học }\end{array}$ & 0.628 & \\
\hline & $\begin{array}{l}\text { Năng lực tìm hiểu về chương trình dạy } \\
\text { học }\end{array}$ & 0.666 & \\
\hline & Năng lực sư phạm thực tiễn & 0.763 & \\
\hline & $\begin{array}{l}\text { Hướng dẫn, hỗ trợ và chia sẻ chuyên môn } \\
\text { bản thân }\end{array}$ & 0.742 & \\
\hline \multirow{5}{*}{$\begin{array}{l}\text { Xây dựng kế hoạch } \\
\text { dạy học }\end{array}$} & $\begin{array}{l}\text { Kế hoạch dạy học trình bày theo quy định } \\
\text { chung }\end{array}$ & 0.556 & \multirow{5}{*}{76.33} \\
\hline & $\begin{array}{l}\text { Xác định mục tiêu dạy học phát triển } \\
\text { phẩm chất, năng lực học sinh }\end{array}$ & 0.762 & \\
\hline & $\begin{array}{l}\text { Nội dung dạy học bám sát chương trình, } \\
\text { hướng tới phát triển phẩm chất, năng lực } \\
\text { học sinh }\end{array}$ & 0.769 & \\
\hline & Hoạt động dạy học gắn với thực tiễn & 0.740 & \\
\hline & $\begin{array}{l}\text { Hướng dẫn, hô̂ trợ và chia sẻ kinh nghiệm } \\
\text { xây dựng kế hoạch dạy học }\end{array}$ & 0.689 & \\
\hline \multirow{2}{*}{$\begin{array}{l}\text { Sử dụng phương } \\
\text { pháp dạy học }\end{array}$} & $\begin{array}{l}\text { Cập nhật được những phương pháp dạy } \\
\text { học tích cực phát triển phẩm chất, năng } \\
\text { lực học sinh }\end{array}$ & 0.766 & \multirow{2}{*}{75.37} \\
\hline & $\begin{array}{l}\text { Vận dụng những phương pháp dạy học } \\
\text { tích cực phát triển phẩm chất, năng lực } \\
\text { học sinh }\end{array}$ & 0.717 & \\
\hline
\end{tabular}




\begin{tabular}{|c|c|c|c|}
\hline & Nhân tố quan sát & $\begin{array}{l}\text { Hệ số tải } \\
\text { nhân tố }\end{array}$ & $\begin{array}{c}\text { Tổng } \\
\text { phương sai } \\
\text { trích } \\
(\%)\end{array}$ \\
\hline & $\begin{array}{l}\text { Phối hợp các phương pháp dạy học tích } \\
\text { cực và các phương pháp dạy học truyền } \\
\text { thống trong một kế hoạch bài dạy }\end{array}$ & 0.770 & \\
\hline & $\begin{array}{l}\text { Hướng dẫn, hỗ trợ và chia sẻ phương pháp } \\
\text { dạy học }\end{array}$ & 0.762 & \\
\hline & $\begin{array}{l}\text { Cập nhật được những hình thức kiểm tra, } \\
\text { đánh giá phát triển phẩm chất, năng lực } \\
\text { học sinh }\end{array}$ & 0.594 & \\
\hline & $\begin{array}{l}\text { Vận dụng được nhiều hình thức kiểm tra, } \\
\text { đánh giá trong kế hoạch dạy học }\end{array}$ & 0.725 & \\
\hline $\begin{array}{l}\text { Kiêm tra, đánh giá } \\
\text { kết quả học tập }\end{array}$ & $\begin{array}{l}\text { Kiểm tra, đánh giá kết quả học tập của học } \\
\text { sinh đảm bảo chính xác, toàn diện }\end{array}$ & 0.708 & 70.99 \\
\hline & $\begin{array}{l}\text { Sử dụng kết quả kiểm tra, đánh giá để điều } \\
\text { chỉnh hoạt động dạy học }\end{array}$ & 0.773 & \\
\hline & $\begin{array}{l}\text { Hướng dẫn, hỗ trợ và chia sẻ kinh nghiệm } \\
\text { kiểm tra, đánh giá kết quả học tập }\end{array}$ & 0.759 & \\
\hline & $\begin{array}{l}\text { Hiểu được phẩm chất, năng lực của học } \\
\text { sinh trong từng lớp học để có hồ trợ kịp } \\
\text { thời }\end{array}$ & 0.596 & \\
\hline Tư vấn và hỗ trọ' & $\begin{array}{l}\text { Thực hiện các phương pháp hỗ trợ trong } \\
\text { quá trình dạy học, xử lí kịp thời các tình } \\
\text { huống phát sinh trong quá trình dạy học }\end{array}$ & 0.776 & 70.94 \\
\hline & $\begin{array}{l}\text { Hố trợ học sinh trong quá trình giải quyết } \\
\text { vấn đề trong kế hoạch dạy học phát triển } \\
\text { phẩm chất, năng lực học sinh }\end{array}$ & 0.755 & \\
\hline & $\begin{array}{l}\text { Chia sẻ kinh nghiệm hỗ trợ học sinh trong } \\
\text { quá trình giảng dạy }\end{array}$ & 0.767 & \\
\hline
\end{tabular}

Nguồn: Kết quả xử lí dữ liệu thống kê từ IBM SPSS Statistic 22.0

$\mathrm{KMO}=0.835$, thỏa mãn điều kiện $0.5 \leq \mathrm{KMO} \leq 01$ cho thấy phân tích nhân tố khám phá trong nghiên cứu này phù hợp với dữ liệu thu được. $\mathrm{Sig}=0.000<0.05$ có nghĩa là các biến quan sát có tương quan tuyến tính với nhân tố đại diện. Kết quả khám phá nhân tố cho thấy các biến quan sát là đại diện của 05 nhân tố trích được từ nhân tố khám phá và trùng khớp với 05 thành phần năng lực đại diện được mô tả từ đầu nghiên cứu. Tổng phương sai trích trong kết quả từ Bảng 3 cho thấy 05 thành phần năng lực của năng lực giảng dạy của sinh viên sư phạm dao động từ 64.05 đến 76.33 các giá trị này đã lớn hơn mức 50\% (Hair, Tatham, et al., 2009). Phát triển chuyên môn bản thân với các biến quan sát trích cô đọng được $65.05 \%$ các nhân tố phân tích đại diện cho thành phần. Tương tự cho xây dựng kế hoạch dạy học (76.33\%), sử dụng phương pháp dạy học (75.37\%), kiểm tra, đánh giá kết quả học tập (70.99\%) và tư vấn hỗ trợ học sinh 
(70.94\%). Hệ số tải nhân tố có giá trị từ 0.501 đến 0.776 có giá trị $\geq 0.5$ cho thấy biến quan sát này có ý nghĩa thống kê tốt. Một lần nữa có thể khẳng định các biến quan sát trong các nhân tố được trích đại diện được cho từng thành phần năng lực cần nghiên cứu trong năng lực giảng dạy của sinh viên sư phạm. 


\subsection{Phân tích các nhân tố quyết định năng lục giảng dạy của sinh viên sư phạm}

Phân tích hồi quy là một phương pháp kiểm định thống kê để phân tích mối quan hệ giữa một biến phụ thuộc duy nhất với một số biến độc lập (nhiều hơn 03) để xem mức độ ảnh hưởng của các biến độc lập đó tác động lên biến phụ thuộc duy nhất như thế nào (McNabb, 2008). Mô hình hồi quy phân tích các thành phần năng lực ảnh hưởng như thế nào đến năng lực giảng dạy chung của sinh viên sư phạm tại Trường Đại học Sư phạm Thành phố Hồ Chí Minh từ góc độ khảo sát sinh viên với các hệ số hồi quy được trình bày ở Bảng 4.

\section{Bảng 4}

Các hệ số hồi quy của các biến về năng lực giảng dạy của sinh viên sư phạm

\begin{tabular}{|c|c|c|c|c|c|}
\hline Nhân tố & Hệ số $\boldsymbol{\beta}$ & Độ lệch chuẩn & Giá trị t & Sig & $\begin{array}{c}\text { Đo lường cộng } \\
\text { tuyê̂n } \\
\text { VIF }\end{array}$ \\
\hline Hằng số & 0.290 & 0.102 & 0.597 & 0.000 & \\
\hline CM & 0.156 & 0.083 & 2.391 & 0.004 & 1.896 \\
\hline KH & 0.444 & 0.060 & 5.463 & 0.000 & 1.136 \\
\hline PP & 0.312 & 0.086 & 4.545 & 0.000 & 1.428 \\
\hline KT & 0.275 & 0.090 & 3.493 & 0.001 & 1.393 \\
\hline HT & 0.120 & 0.080 & 2.560 & 0.000 & 1.885 \\
\hline
\end{tabular}

Nguồn: Kết quả xử lí dữ liệu thống kê từ IBM SPSS Statistic 22.0

Kết quả cho thấy giá trị kiểm định với mức ý nghĩa Sig. $=0.000<0.05$. Điều này chứng tỏ rằng mô hình hồi quy xây dựng là phù hợp với bộ dữ liệu thu thập được. 05 thành phần năng lực được mô tả đều có ý nghĩa trong mô hình nghiên cứu và có tác động có ý nghĩa đến năng lực giảng dạy của sinh viên sư phạm tại Trường Đại học Sư phạm Thành phố Hồ Chí Minh. Giá trị $\mathrm{R}^{2}$ hiệu chỉnh của hồi quy bằng 0.885 cho thấy các biến độc lập là các năng lực thành phần $(05$ năng lực thành phần) đưa vào mô hình giải thích được $88.5 \%$ sự thay đổi của biến phụ thuộc. Cho thấy phát triển chuyên môn bản thân, xây dựng kế hoạch dạy học, sử dụng phương pháp dạy học, kiểm tra đánh giá kết quả học tập và tư vấn hỗ trợ học sinh chiếm $88.5 \%$ việc hình thành năng lực giảng dạy của sinh viên sư phạm tại Trường Đại học Sư phạm Thành phố Hồ Chí Minh. Giá trị VIF trong hồi quy mô tả hiện tượng đa cộng tuyến. Mô hình nghiên cứu sử dụng bảng hỏi 05 mức độ với VIF < 2.000 sẽ không có hiện tượng đa cộng tuyến xảy ra. Đa cộng tuyến là hiện tượng các biến độc lập có mối tương quan rất mạnh với nhau. Mô hình hồi quy xảy ra hiện tượng đa cộng tuyến sẽ khiến nhiều chỉ số bị sai lệch, dẫn đến kết quả của việc phân tích hồi quy tuyến tính không còn mang lại nhiều ý nghĩa. Trong kết quả nghiên cứu, chỉ số VIF của các biến độc lập có giá trị từ 1.136 đến 1.896 đều bé hơn 2.000 , do đó cấu trúc năng lực giảng dạy trong nghiên cứu này không có hiện tượng đa cộng tuyến xảy ra.

Phương trình hồi quy có dạng:

$$
\mathrm{NLGD}=\beta_{1} * \mathrm{CM}+\beta_{2} * \mathrm{KH}+\beta_{3} * \mathrm{PP}+\beta_{4} * \mathrm{KT}+\beta_{5} * \mathrm{HT}+\mathrm{e}
$$

Với: NLGD: "năng lực giảng dạy"; CM: "Phát triển chuyên môn bản thân"; KH: "Xây dựng kế hoạch dạy học"; PP: "Sử dụng phương pháp dạy học"; KT: "Kiểm tra, đánh giá kết quả học tập"; HT: "Tư vấn và hỗ trợ học sinh"; e: hằng số $(\mathrm{e}=0.29)$.

Hằng số và các biến độc lập $(\mathrm{CM}, \mathrm{KH}, \mathrm{PP}, \mathrm{KT}, \mathrm{HT})$ đều có giá trị $\mathrm{Sig}<0.05$ có ý nghĩa về mặt thống kê trong phương trình hồi quy. Trong nghiên cứu, sau khi có kết quả hồi quy ta có 
02 hệ số beta làm giá trị " $\beta$ ” của các biến, tuy nhiên ta sử dụng hệ số beta đã chuẩn hóa để có phương trình hồi quy dạng chuẩn hóa tin cậy nhất. Như vậy năng lực giảng dạy của sinh viên được thể hiện ở phương trình hổi quy sau:

$$
\mathrm{NLGD}=0.444 * \mathrm{KH}+0.312 * \mathrm{PP}+0.275 * \mathrm{KT}+0.156 * \mathrm{CM}+0.120 * \mathrm{HT}+0.290
$$

Từ các kết quả phân tích trên, mô hình nghiên cứu giả định ban đầu đã phù hợp và đã được chứng minh. Các thành phần năng lực và các biến quan sát phù hợp để cấu trúc thành năng lực giảng dạy của sinh viên sư phạm tại Trường Đại học Sư phạm Thành phố Hồ Chí Minh. Hình 1 sau thể hiện cấu trúc năng lực giảng dạy của sinh viên sư phạm với các năng lực thành phần và mức độ tác động.

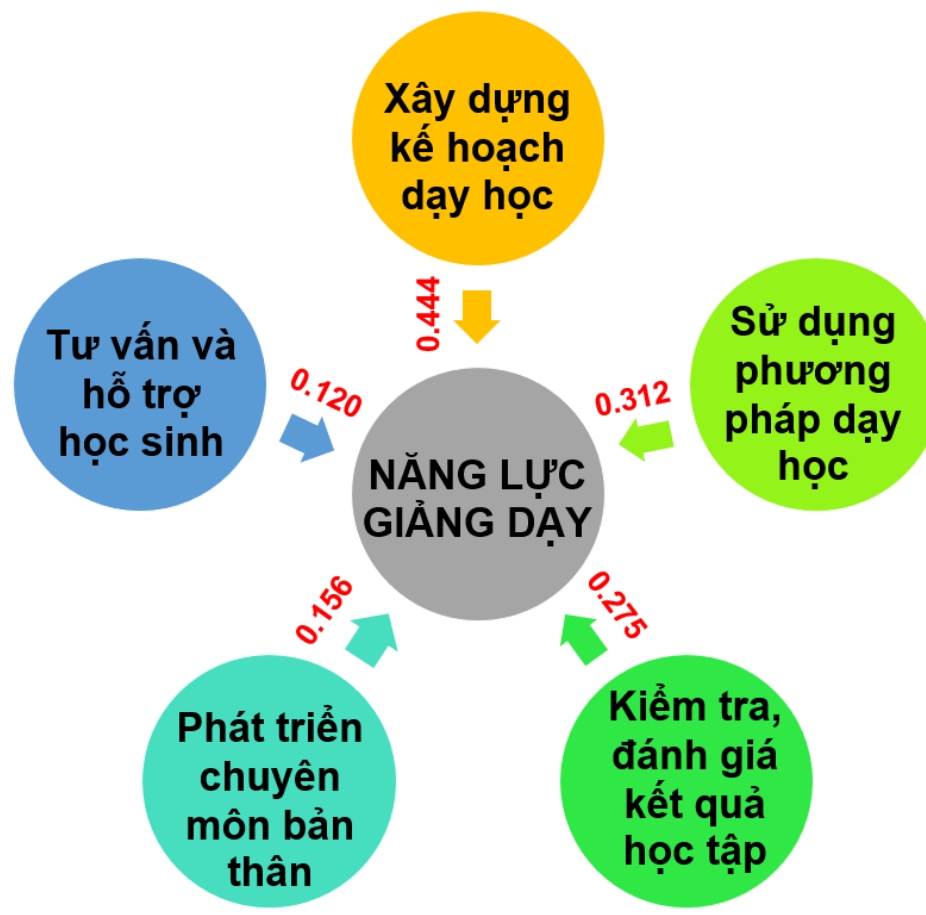

Hình 1. Mô hình cấu trúc năng lực giảng dạy của sinh viên sư phạm

Kết quả phân tích cho thấy 05 nhân tố khảo sát đều có mức ý nghĩa $1 \%(\mathrm{Sig} \leq 0.01)$ có tác động cùng chiều đến năng lực giảng dạy của sinh viên sư phạm bao gồm: KH: "Xây dựng kế hoạch dạy học"; PP: "Sử dụng phương pháp dạy học"; KT: "Kiểm tra, đánh giá kết quả học tập"; CM: "Phát triển chuyên môn bản thân", HT: "Tư vấn và hỗ trợ học sinh". Kết quả cho thấy xây dựng kế hoạch dạy học có tác động lớn nhất đến năng lực giảng dạy của sinh viên sư phạm với $\beta$ $=0.450$. Tiếp đến lần lượt là sử dụng phương pháp dạy học $(\beta=0.312)$, kiểm tra, đánh giá kêt quả học tập $(\beta=0.272)$, phát triển chuyên môn bản thân $(\beta=0.154)$. Nội dung tư vấn và hỗ trợ học $\sinh (\beta=0.128)$ có tác động thấp nhất đến năng lực giảng dạy của sinh viên sư phạm.

\section{Kết luận và gợi ý}

Năng lực giảng dạy là năng lực cơ bản và quan trọng nhất đối với người dạy. Sinh viên sư phạm là những người giảng dạy trở thành giáo viên trong tương lai vì thế cần tiếp cận tiêu chuẩn năng lực giảng dạy. Cấu trúc năng lực giảng dạy trong nghiên cứu bao gồm các thành tố: xây dựng kế hoạch dạy học, sử dụng phương pháp dạy học, kiểm tra, đánh giá kết quả học tập, phát triển chuyên môn bản thân, tư vấn và hỗ trợ học sinh với các mức độ tác động khác nhau tạo thành năng lực giảng dạy đối với sinh viên sư phạm. Tuy nhiên, các thành tố này chỉ mới mô tả được $88.5 \%$ năng lực giảng dạy của sinh viên sư phạm vì thế cần có thêm các nghiên cứu xác định các thành tố còn có thể tác động đến năng lực giảng dạy của sinh viên sư phạm. Trong nghiên cứu này còn hạn chế khi chỉ đánh giá thông qua các sinh viên sư phạm của các ngành 
thuộc khoa học tự nhiên (sư phạm Vật lý, sư phạm Hóa học và sư phạm Sinh học), vì thế trong những nghiên cứu tiếp theo có thể mở rộng nghiên cứu ra các ngành khác. Thông qua cấu trúc năng lực giảng dạy này là cơ sở để sinh viên sư phạm rèn luyện khả năng giảng dạy của bản thân cũng như các trường đại học đào tạo giáo viên căn cứ làm cơ sở có những hoạch định trong đào tạo trong tương lai. Các trường đại học sư phạm cần chú trọng và phân bố thời gian rèn luyện tương ứng với mức độ tác động của các năng lực giảng dạy như trên.

\section{Tài liệu tham khảo}

Ban chấp hành Trung ương Đảng Cộng sản Việt Nam. (2013). Nghị quyết số 29/NQ-TW ngày 04 tháng 11 năm 2013 Hội nghi lần thư Tám Ban chấp hành Trung ương khóa XI về đổi mới căn bản, toàn diện giáo dục và đào tạo [Resolution No. 29/NQ-TW dated November 4, 2013 The 8th Conference of the XI Central Executive Committee on fundamental and comprehensive innovation of education and training]. Retrieved May 10, 2021, from https://moet.gov.vn/tintuc/Pages/doi-moi-can-ban-toan-dien-gd-va-dt.aspx ?ItemID=3928

Ban chấp hành Trung ương Đảng Cộng sản Việt Nam. (2021). Nghị quyết ngày 01 tháng 02 năm 2021 Đại hộ đại biểu toàn quốc lần thứ XIII của Đảng [Resolution February 1, 2021 of 13th National Delegated Congress of the Communist Party]. Retrieved May 10, 2021, from https://daihoi13.dangcongsan.vn/tu-lieu/van-ban-chi-dao-huong-dan

Bộ Giáo dục và Đào tạo. (2018a). Thông tu 32/2018/TT-BGDĐT ngày 26 tháng 12 năm 2018 về Chuơng trình giáo dục phổ thông - Chuoong trình tổng thể [Circular 32/2018/TT-BGDDT dated December 26, 2018 on General education curriculum - overall curriculum]. Retrieved May 10, 2021, from https://moet.gov.vn/van-ban/vanban/Pages/chi-tiet-vanban.aspx?ItemID=1301

Bộ Giáo dục và Đào tạo. (2018b). Thông tu số 20/2018/TT-BGDĐT ngày 22 tháng 8 năm 2018 về ban hành quy định chuẩn nghề nghiệp giáo viên co sở giáo dục phổ thông [Circular No. 20/2018/TT-BGDDT dated August 22, 2018 on Promulgate professional standards for teachers at general education institutions]. Retrieved May 10, 2021, from https://moet.gov.vn/van-ban/vanban/Pages/chi-tiet-van-ban.aspx?ItemID=1290

Bui, D. M., Dao, A. T. V., \& Hoang, H. T. K. (2012). Đổi mới mô hình đào tạo giáo viên trong các trường Đại học Sư phạm theo hướng tiếp cận năng lực [Renovating the model of teacher training in pedagogical universities in the direction of competency approach]. Tạp chí Giáo dục, 277(1), 2-5.

Cronbach, L. J. (1951). Coefficient alpha and the internal structure of tests. Psychometrika, 16(3), 297-334.

Dinh, B. Q. (2013). Phẩm chất nghề nghiệp và định hướng đào tạo giáo viên đáp ứng yêu cầu đổi mới giáo dục phổ thông [The professional quality and teacher training orientation respones the requirements of education]. Tạp chí Giáo duc, 307(1), 4-6.

Fielding, G. D., \& Schalock, H. D. (1985). Promoting the professional development of teachers and administrators (ERIC/CEM School Management Digest Series, No. 31). Oregon Eugene, Oregon: College of Education University of Oregon Eugene.

Gebre, E., Saroyan, A., \& Bracewell, R. (2014). Students' engagement in technology rich classrooms and its relationship to professors' conceptions of effective teaching. British Journal of Educational Technology, 45(1), 63-96.

Hair, J. F., Black, W. C., Babin, B. J., \& Anderson, R. E. (2009). Multivariate data analysis: A 
global perspective (7th ed.). New York, NY: Prentice Hall.

Hair, J. F., Tatham, R. L., Anderson, R. E., \& Black, W. (2009). Multivariate data analysis (6th ed.). Upper Saddle River, NJ: Prentice Hall.

Hammond, L. D. (2006). Constructing 21st-century teacher education. Journal of Teacher Education, 57(3), 300-314.

Le, C. M. (2019). Đổi mới công tác bồi dương năng lực dạy học cho giáo viên phổ thông [The issue of innovation on fostering teaching competency for general school teachers]. Tạp chí Giáo dục, 5(2), 33-36.

McNabb, D. E. (2008). Research methods in public administration and nonprofit management: Quantitative and qualitative approaches (2nd ed.). Armonk, NY: M.E. Sharpe.

Mirzagitova, A. L., \& Akhmetov, L. G. (2015). Self-development of pedagogical competence of future teacher. International Education Studies, 8(3), 114-121.

Nguyen, D. T. (2013). Giải pháp nâng cao chất lượng kĩ năng nghề nghiệp cho sinh viên trường Cao đẳng Sư phạm Lạng Sơn [Solutions to improve the quality of professional skills for students of Lang Son College of Pedagogy]. Tạp chí Giáo dục, 314(2), 22-24.

Nguyen, H. T. T. (2015). Hình thành năng lực dạy học cho sinh viên Đại học Sư phạm qua giảng dạy môn giáo dục học đáp ứng yêu cầu đổi mới giáo dục - đào tạo [Establishing teaching capacity for educational students through teaching Education to meet the requirements of education and training innovation]. Tạp chí Khoa học Truò̀ng Đại học Su phạm Hà Nội, 60(8B), 172-179.

Nunnally, J. (1978). Psychometric theory. New York, NY: McGraw-Hill.

Panagiota, V. S., \& Eleni, K. S. (2021). Instructional effectiveness of elementary education university students: Innovative approaches to learning through the learning theory of Biopedagogism. International Journal of Learning in Higher Education, 28(1), 113-126.

Shulman, L. S. (1987). Knowledge and teaching: Foundations of the new reform. Harvard Educational Review, 57(1), 1-23.

Trinh, G. T., \& Mai, K. Q. (2019). Đào tạo năng lực dạy học cho sinh viên đại học sư phạm theo tiếp cận năng lực thực hiện đáp ứng yêu cầu đổi mới giáo dục phổ thông [Training teaching cpmpetence for teacher students of education universities based on conpetence approach to meet the requirements of general education reforms]. Tap chí Khoa hoc Giáo dục Việt Nam, 22, 34-39.

Voss, T., Kunter, M., \& Baumert, J. (2011). Assessing teacher candidates' general pedagogical/psychological knowledge: Test construction and validation. Journal of Educational Psychology, 103(4), 952-969.

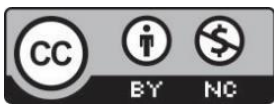

Creative Commons Attribution-NonCommercial 4.0 International License. 